\title{
L'histoire des sciences au seuil du troisième millénaire
}

Des hommes, des idées et des institutions*

\section{R. Halleux}

L'histoire conceptuelle de l'histoire des sciences a été souvent racontée. Il n'en va pas de même pour son histoire institutionnelle. Elle vaut cependant d'être méditée, surtout dans les périodes de crise, car la naissance des institutions, leur développement, leur sclérose et leur mort sont d'abord le fait des hommes et des idées. Dans cette histoire, trois phases, dont deux aprèsguerre:

- dans les années 20, la naissance de l'Académie internationale d'histoire des sciences

- dans les années 45-50, l'histoire des sciences à l'UNESCO et la naissance de l'Union internationale

- dans la dernière décennie, la multipolarisation des réseaux, et l'émergence de nouvelles problématiques.

A simplifier grossièrement, on pourrait dire que l'organisation internationale de notre discipline est le fait de deux hommes, George Sarton et Aldo Mieli, tous deux animés d'un humanisme mondialiste bien dans l'esprit de la Société des Nations et de l'Institut International de Coopération intellectuelle.

George Sarton, un physicien belge établi aux Etats-Unis, écrivait dans sa revue Isis: «History of Science is the history of mankind.» Après la guerre, on pouvait en effet considérer la science comme le seul processus universel et cumulatif dans l'histoire de l'humanité. Générant ses propres valeurs, elle

* Dr. Markus Guggenheim-Schnurr-Gedenkvortrag, gehalten anlässlich der Jahrestagung der Schweizerischen Gesellschaft für Geschichte der Medizin und der Naturwissenschaften in Luzern am 14. Oktober 1999.

Prof. R. Halleux, Secrétaire Général de l'Union Internationale, d'Histoire et de Philosophie des Sciences, Centre d'Histoire des Sciences et des Techniques, Université de Liège, 15 av. des Tilleuls, B-4000 Liège. 
devenait la base d'un nouvel humanisme. La correspondance inédite de Sarton, conservée au Mundaneum de Mons, contient le projet d'un Institut international d'Histoire des Sciences, pour lequel il sollicita en vain les mécènes américains.

Aldo Mieli, chimiste influencé par Duhem, Mach et Sarton, partageait avec ce dernier le rêve d'universalité et d'unité. Dès son plus jeune âge, il rêvait «d'arriver avec peu de prémisses à une compréhension universelle de tous les phénomènes physiques et sociaux, artistiques et philosophiques». Aussi essaiera-t-il, à diverses reprises, en 1916, 1925, 1935, 1939, d'écrire une histoire générale des sciences qui n'ira jamais plus loin que le premier volume. En 1919, il lance une revue, Archeion, qui reflète le même idéal mondialiste. Typiques de cet état d'esprit, les sommaires en interlingua, une langue synthétique créée par le logicien Peano.

En 1928, Mieli, antifasciste, dut quitter l'Italie pour la France où Henri Berr lui offrit l'hospitalité du Centre international de Synthèse. Peu de rencontres intellectuelles furent aussi fécondes pour le décloisonnement de l'histoire des sciences. En 1919, Henri Berr (1863-1954) avait lancé la Bibliothèque de Synthèse Historique et la série l'Evolution de l'Humanité, histoire universelle en cent volumes. En 1921, la Revue de Synthèse Historique fondée en 1900 devenait Revue de Synthèse. En 1925, Henri Berr créait le Centre International de Synthèse comme pendant de l'Ecole Pratique des Hautes Etudes, vouée à l'analyse. Le Centre s'installait d'abord dans les locaux de l'Institut International de Coopération Intellectuelle, puis à partir de 1929 au 12 de la Rue Colbert, dans l'ancien hôtel de Nevers. Le Centre comportait une section d'Histoire des Sciences, dont Mieli fut directeur et Archeion l'organe.

En 1928, à l'occasion du Congrès International des Sciences Historiques à Oslo, Mieli crée avec Abel Rey, George Sarton, Henry Sigerist, Charles Singer, Karl Sudhoff, Lynn Thorndike un Comité International d'Histoire des Sciences qui organise à Paris en 1929 son premier congrès international. Le Comité deviendra l'Académie Internationale d'Histoire des Sciences et Archeion son organe officiel. L'Académie, combinant association de personnes et représentations nationales, créera très tôt des groupes nationaux: Bolivie, Chili, Equateur, Inde, Mexique, Philippines, Pérou, Turquie, etc. ancrant ainsi dans l'institutionnel une histoire des sciences ouverte au monde entier.

Les congrès de Londres (1931), Coimbra (1934), Prague (1937) sont le fruit de ce dynamisme. Il faut lire les chroniques d'Archeion pour percevoir l'enthousiasme des fondateurs et la beauté de leur utopie. L'essor fut cassé 
par la guerre. Mieli, réfugié en Argentine, s'efforça de maintenir Archeion et mourut dans la misère.

La deuxième période s'ouvre avec la création de l'UNESCO. Dans l'euphorie de la victoire du «monde libre», l'UNESCO avait proposé de rechercher «dans quelle mesure les différentes écoles philosophiques et les diverses églises peuvent arriver à un accord quant aux principes généraux, conformes à l'état actuel des sciences, sur lesquels il serait possible de fonder un monde spirituellement uni».

Pour réaliser cette tâche démesurée, l'UNESCO se donna deux moyens:

- un grand projet d'écriture de l'histoire du développement scientifique et culturel de l'humanité

- un réseau d'organisations non gouvernementales dont une Union internationale d'histoire et de philosophie des sciences.

\section{L'entreprise historiographique}

Dès 1946, le biologiste Julian Huxley, secrétaire exécutif de la commission préparatoire pour l'UNESCO, lança l'idée d'une «Histoire mondiale du développement des sciences et des cultures». La résolution fut approuvée à la deuxième session de la conférence générale à Mexico City en 1947, et reprise à la troisième session, tenue à Beyrouth en 1948 (résolution S. 7).

Il appartenait au fondateur des Annales, Lucien Febvre, de renouer avec le rêve mondialiste d'Henri Berr et d'Aldo Mieli. A la première conférence générale, il proposait d'écrire désormais le mot civilisation au pluriel. En 1949, devant le Conseil International de la Philosophie et des Sciences Humaines, il esquissait le plan d'un ouvrage qui «prétend agir sur les mentalités en extirpant le mortel virus de la guerre». Toutes les grandes questions y sont rencontrées. Ainsi le volume I serait consacré aux questions de base relevant de l'anthropologie et de la biologie humaine, de l'ethnologie, de la psychologie et de la linguistique. En conclusion, viendrait

une grosse question: dans leur développement les divers groupes humains suivent-ils la même marche, accomplissent-ils les mêmes étapes? Les groupes que nous trouvons aujourd'hui à un certain stade de développement, à quelle condition pourraient-ils, renonçant à leur stagnation, poursuivre leur marche pour rattraper les groupes qui les ont dépassés? Doiventils, pour le faire, abandonner leurs idées propres et emprunter celles des groupes plus avancés qu'eux?

Les volumes II et III devraient être consacrés «à des études analytiques sur les échanges. De tout cela se dégagerait l'image d'une humanité mouvante dès l'origine, et se déplaçant sans cesse au cours d'une série perpétuelle de 
migrations transcontinentales.» Quant aux volumes IV et V, ils opéreraient un regroupement synthétique dans le cadre géographique.

Tour à tour ils examineraient ce que l'Asie, les hommes d'Asie doivent à l'Europe, à l'Afrique, à l'Océanie, à l'Amérique dans tous les domaines et à toutes les époques. Ensuite ce que l'Europe et les Européens ont donné et reçu, ce que l'Afrique et les Africains, l'Amérique et les Américains, l'Océanie et les Océaniens ont reçu des autres parties du monde et leur ont en échange donné. De ce tableau se dégagerait l'idée que le cloisonnement du monde n'est qu'une fiction et que la terre n'a cessé de se diversifier, de s'enrichir, de se féconder par un flot d'échanges pacifiques.

Dans ce projet ambitieux de première «histoire pacifique de l'humanité», les sciences et techniques de toutes les aires culturelles avaient leur place.

Il ne fut jamais appliqué. Le 23 août 1949, Miguel Ozorio de Almeida présentait au Secrétaire Général un rapport reflétant des positions, d'emblée peu conciliables, d'une Histoire Scientifique et culturelle de l'Humanité où les idées-forces de Lucien Febvre se trouvaient noyées dans un plan de compromis d'inspiration nettement positiviste.

La présidence de la Commission internationale pour une histoire du développement scientifique et culturelle de l'humanité fut confiée à un membre éminent de l'Eglise positiviste brésilienne, Paulo E. de Barrêdo Carneiro. Lucien Febvre, quant à lui, se voyait confier la direction des Cahiers d'Histoire Mondiale.

Les deux entreprises ont connu des destinées assez différentes. Le premier volume de History of Mankind. Cultural and scientific development parut en 1963. La préface de René Maheu, directeur général de l’UNESCO, montrait à suffisance qu'il s'agissait d'une œuvre de compromis:

The History is the work, not of a team with a homogeneous cultural background, but of an international Commission which by its very composition and even more by the spirit pervading it, embraces all the varied cultural traditions and modern ideologies which form the spiritual framework of our present day world.

On ne pouvait mieux résumer les tensions entre historiographie marxiste et non marxiste, laïque et religieuse dont plusieurs participants ont gardé le souvenir. Malgré la «décentralisation des points de vue et des interprétations» le compromis de base était loin des anticipations audacieuses de Lucien Febvre. Ecarté en principe, le point de vue européocentriste dominait en fait, à travers la vision occidentale des sciences et techniques, commune aux capitalistes et aux marxistes.

Les Cahiers d'Histoire Mondiale, quant à eux, engrangèrent depuis 1953, sous l'impulsion de Lucien Febvre et de ses successeurs des matériaux de haute qualité. L'esprit des Annales y est partout présent avec études structurelles et comparées, études de contact et de diffusion, mais peu de ces matériaux trouvaient leur voie jusqu'à la synthèse finale. 
Du reste, le gros des historiens professionnels des sciences ont boudé l'entreprise de I'Unesco et préféré le confort de la dernière entreprise positiviste, l'Histoire Générale des Sciences de René Taton, universelle d'intention, européenne et positiviste par nécessité, faute d'avoir pu assurer un recrutement d'auteurs assez diversifié.

\section{L'Union Internationale}

Par ailleurs, l'UNESCO s'entoura d'organisations non gouvernementales, le Conseil International de la Philosophie et des Sciences humaines (1949) et le Conseil International des Unions scientifiques, déjà fondé en 1931. C'est semble-t-il, Joseph Needham qui eut l'idée d'introduire l'histoire des sciences au sein des Unions scientifiques sous la forme d'une Union spécialisée. Cette Union devait grouper des comités nationaux reconnus par leurs pays respectifs. Ce propos aboutissait à dépouiller l'Académie de sa représentativité internationale et à la réduire à une association de personnes. Les négociations furent menées, pour l'Unesco, par Joseph Needham (UK) et Armando Cortesao (Portugal), pour l'Académie, par Pierre Sergescu (Roumanie), Arnold Reymond (Suisse) J. A. Vollgraff (Pays-Bas), et Pierre Brunet (France). Au $5^{\mathrm{c}}$ congrès, à Lausanne, l'Union vit le jour et l'Académie était reconnue comme «organe d'orientation de son activité scientifique».

Peu à peu distendus, les rapports entre l'Union et l'Académie furent totalement rompus en 1971. Désormais fondée sur la sociabilité des personnes et alignée sur d'autres compagnies savantes, l'Académie se donna pour tâche de soutenir l'histoire des sciences par des moyens plus modestes, mais efficaces: cooptation de savants distingués, médaille Koyré, prix des jeunes historiens, publication de la revue Archives Internationales d'Histoire des Sciences et de la Collection de Travaux, sans oublier la conférence solennelle qu'un membre de l'Académie fait à chaque congrès international d'Histoire des Sciences.

Quant à l'Union, bien des obstacles l'empêchèrent de donner sa pleine mesure. Dans certaines traditions nationales, comme en France l'Histoire des Sciences est étroitement liée à la philosophie, mais cette liaison n'est ni obligatoire, ni universelle. Les philosophes des sciences avaient, dès 1949, créé leur propre Union qui ne put franchir la porte de l'ICSU. Au terme d'un long travail d'infiltration, un ultimatum de l'ICSU le 30 mars 1955, contraignait les historiens à former avec les philosophes une seule Union. Ce mariage forcé fut consommé en 1956. Historiens et philosophes furent souvent bien près du divorce. C'est seulement en août 1999 que, grâce aux 
efforts d'Erwin Neuenschwander, Président de la Commission conjointe, un protocole de collaboration fut signé entre les deux divisions (historiens et philosophes). La prochaine conférence conjointe sur la notion de modèle a eu lieu à Zurich en 2000.

Ces difficultés ne furent pas les seules: un équilibre complexe et paralysant entre Est et Ouest, un manque chronique d'argent, et souvent, il faut le dire, des officiers plus soucieux d'honneurs et de dignités que du bien commun.

Les réalisations ne sont pas pour autant négligeables: des congrès internationaux tous les quatre ans; douze commissions dont cinq inter-unions, cinq groupes spécialisés, 44 membres nationaux.

Telles sont les institutions qui affrontent à présent les bouleversements du paysage scientifique international au seuil du troisième millénaire. L'effondrement du monde communiste permet, en théorie, d'édifier l'histoire des sciences multipolaire dont rêvaient Sarton et Mieli. En pratique, la crise économique mondiale concentre la décision aux mains des pays du Nord. Mais la discipline elle-même se modifie profondément.

De l'observatoire privilégié que constitue la position de Secrétaire Général, j'aimerais, dans une dernière partie, analyser quelques-unes de ces mutations :

1) le déplacement du centre de gravité de la recherche vers la période contemporaine; 2) le triomphe de l'approche externaliste et du socialconstructivisme; 3) la réappropriation des histoires nationales, corrélative à la fin de l'antagonisme est-ouest; 4) l'avancée considérable des sciences non européennes; 5) l'ambiguïté de la situation institutionnelle.

\section{Le triomphe de l'histoire la plus contemporaine}

Jusque dans les années 60, c'est la science classique, de Copernic à Lavoisier, qui attirait le gros des publications, des thèses et des charges d'enseignement. Depuis lors, l'intérêt s'est concentré sur le XIX ${ }^{\mathrm{c}}$ et surtout le $\mathrm{XX}^{\mathrm{c}}$ siècle, particulièrement en physique et en biologie fondamentale. Il est vrai que dans ce domaine, le siècle qui s'achève a connu plus de révolutions que tous les autres ensemble. Mais ce champ nouveau pose des problèmes inédits de documentation et de critique. En documentation, il importe de préserver les témoins de la science qui se fait: papiers, instruments, archives électroniques. Mais que garder, que jeter dans la masse immense générée par la science contemporaine? C'est la tâche principale de la Commission «bibliographie et documentation», qui a organisé deux workshops en 1996 et 1997. Au 
prochain congrès, on se donnera pour but d'élaborer, avec le conseil international des Archives un World Directory of Scientists Papers. Il en va de même pour les instruments. Pour les instruments de haute époque, la valeur commerciale garantit leur préservation. Mais qui se soucie de conserver un vieil ordinateur, avec ses logiciels et ses pièces détachées? Et pourtant, c'est une priorité si on veut que la science d'aujourd'hui soit accessible aux historiens de demain. Et cela suppose une politique concertée de préservation, en tout cas au niveau européen, un cadastre des machines, de manière qu'une pièce ne soit conservée qu'une fois. Par ailleurs, l'absence de recul historique nécessite de préciser ou d'affirmer les méthodes critiques d'une «histoire immédiate».

\section{De l'externalisme au social-constructivisme}

Née dans la mouvance positiviste, l'histoire des sciences a longtemps privilégié l'étude des mécanismes internes d'évolution du savoir. De ce fait, elle n'a pas peu contribué à forger la conscience identitaire de la communauté scientifique. Face à cette approche internaliste s'est développée, après la guerre, une approche externaliste, qui prend de plus en plus en compte le rôle des facteurs institutionnels, politiques, économiques et sociaux.

Lointainement, la problématique s'enracine dans le matérialisme historique de Friedrich Engels dans l'Anti-Dühring. D'autre part, Lucien Febvre et l'école des Annales ont élargi le territoire de l'historien à l'histoire des sciences, en privilégiant les rapports avec la technique, l'étude des communautés, des institutions, de la communication et du livre, des visions du monde et des mentalités.

Mais il en va tout autrement d'un sociologisme radical, né de la contestation du rôle politique de la science contemporaine de l'après-guerre, et lié au courant que l'on appelle aujourd'hui postmoderniste. La rapide diffusion de cette approche externaliste et sociologique est allée de pair avec le rejet des valeurs de la raison en particulier des Lumières après la Shoah et les bombes atomiques sur le Japon. Elle a subi profondément l'influence de l'approche marxiste de Bernal et de Needham et s'est principalement enracinée dans le monde anglo-saxon marqué par la concomitance entre la Révolution scientifique et la Révolution d'Angleterre.

C'est spécialement l'école américaine, née des travaux de Robert Merton, qui a ainsi transformé l'histoire sociale de la science en sociologisme sectaire. A sa suite, les post-kuhniens ont cru pouvoir établir que le changement de paradigme était dicté par les attitudes de la communauté scientifique. 
En associant la vérité scientifique au consensus entre les savants, ils ont introduit en histoire des sciences un relativisme qui gomme la spécificité du projet scientifique en tant que recherche de la vérité.

Puisqu'un changement de paradigme est pure question de consensus social, la science est un produit social comme tant d'autres. Comme l'écrit Isabelle Stengers, la science est ce qui, à une époque donnée, est pratiqué par les gens qui s'appellent eux-mêmes savants. A force de présenter la science comme un phénomène humain trop humain, les historiens postmodernes se posent ainsi en alliés objectifs de ceux qui veulent réintroduire subrepticement la puissance du divin et du discours théologique. Quelques mots de Henri Berr tirés de la Synthèse en histoire (1911) méritent d'être ici rappelés:

Jamais la pensée n'a été totalement indépendante du milieu social - de même qu'elle n'a jamais été à l'abri des contingences de toutes sortes: et ces influences sont à déterminer. Mais ce qu'il importe aussi et surtout de préciser, c'est le travail interne de la pensée. Il faut suivre l'évolution qui s'accomplit dans ce milieu - différent du milieu social - que constituent les hommes en tant qu'êtres pensants.

En fait, le sociologisme simplificateur méconnait que le contexte social définit les conditions de possibilité et non d'existence.

Toutefois, en investissant l'histoire des sciences avec les armes de l'histoire sociale, l'approche externaliste a grandement contribué à intégrer l'histoire des sciences au mouvement de l'histoire générale.

\section{Le renouveau des histoires nationales}

L'écriture des histoires nationales des sciences était, au XIX ${ }^{\mathrm{e}}$ siècle, un pur produit du chauvinisme. La galerie des grands hommes, puissant aliment de la fierté nationale, fut la première tâche des nations, surtout des jeunes. La revendication de nationalité, la querelle de priorité, en furent les corollaires. D'où les débats sur la nationalité de Copernic ou sur l'originalité des découvertes de Lavoisier, ou sur l'apport des savants russes. Après l'effondrement du bloc communiste, cet intérêt pour les racines nationales a pris une autre forme.

En histoire des sciences comme ailleurs, c'est la politique qui écrit l'histoire. Dans les pays anciennement occupés par l'ancien Soviétique, l'affirmation de l'autonomie politique va de pair avec la redécouverte de l'histoire nationale ou régionale des sciences et la délimitation de nouveaux espaces culturels. C'est le sens de l'action que mène en Lituanie, Lettonie, Estonie, l'association Baltique d'Histoire des Sciences. Les anciennes républiques russes d'Asie centrale s'efforcent fièvreusement de ressusciter leur 
passé islamique. De même en Turquie, les recherches sur la science dans l'ancien espace ottoman comme interface entre l'Est et l'Ouest transposent dans le passé les débats sur la vocation culturelle et politique de la Turquie d'aujourd'hui. Les dérives nationalistes sont en germe et on fera bien de se souvenir que la science opère toujours dans un espace à géométrie variable. Les diverses institutions qui œuvrent à la construction de l'identité européenne recourent semblablement à l'histoire des sciences. La méthode expérimentale et les droits de l'homme, répète-t-on, sont le ciment de notre unité.

\section{L'étude des sciences non occidentales}

A une échelle bien plus large, l'histoire de la science indienne, de la science chinoise, de la science arabo-islamique, échappent de plus en plus aux historiens occidentaux. En terre d'Islam, les historiens replacent la science dans son contexte religieux dont on l'avait artificiellement arrachée. Enfin, les civilisations du Sud, à tradition orale, sont aussi exclues de l'historiographie des sciences que de la science d'aujourd'hui, réclamant une place dans le paysage mondial du savoir. A la World Conference on science, organisée en juin 1999 à Budapest par l'ICSU et l'UNESCO, le sud exigeait une section sur Beyond science, Science and other systems of knowledge. Organisée par notre Union, la session conclut à la nécessité de revaloriser les connaissances traditionnelles. Je cite le $\S 26$ de la résolution finale

que les savoirs traditionnels et locaux, qui sont l'expression dynamique d'une certaine perception et compréhension du monde, peuvent, et historiquement ont apporté, une précieuse contribution à la science et à la technologie et qu'il faut absolument préserver, protéger, promouvoir et étudier ce patrimoine culturel et ces connaissances empiriques.

Deux mois plus tard, l'assemblée générale de l'ICSU, réunie au Caire, entérinait presque toutes les conclusions de la conférence, sauf celle-là. Le champ d'étude se trouvait limité aux connaissances empiriques découvertes par accident. La tâche est désormais prise en charge par une commission spécialisée de notre Union.

La question majeure, que l'on ne peut esquiver, est la fameuse Needham question: pourquoi la Révolution Scientifique s'est-elle faite en Europe, alors que les autres cultures avaient tous les atouts pour la réussir? Il apparaît de plus en plus que l'histoire des sciences, au sens ancien, devra un jour où l'autre se réécrire en histoire transculturelle des savoirs.

Dans cette même perspective, l'étude des transferts de savoir entre les métropoles et les colonies s'inscrit à présent dans la problématique globale que l'on appelle généralement science and empire. En Inde, en Amérique 
Latine et en Afrique, on analyse non seulement le savoir colonial, mais le choc en retour sur les métropoles et les spécificités résultant du métissage des cultures.

\section{Une position institutionnelle ambiguë}

En un siècle, la recherche en histoire des sciences est devenue florissante. Les praticiens se sont professionnalisés. Bien loin de l'universalisme de Sarton et de Mieli, ils se sont spécialisés, comme les scientifiques eux-mêmes, dans un domaine souvent étroit. Certains vont jusqu'à envisager la suppression des congrès internationaux ou leur transformation en collection de colloques spécialisés. Or, nos partenaires, scientifiques et historiens, attendent de nous, outre la connaissance de notre spécialité, une culture généraliste que l'on ne peut acquérir que dans ces contextes interdisciplinaires.

Car la situation académique de notre discipline est précaire. L'essor de la recherche contraste avec une véritable marginalité institutionnelle, particulièrement dans l'enseignement.

Du 25 au 28 juin 1998, le groupe ALLEA, All European Academies, c'està-dire l'ensemble des Académies des Sciences d'Europe, organisait à Strasbourg une ample conférence sur le thème History of Science and Technology in Education and Training in Europe. Elle a révélé un tableau assez paradoxal: une recherche très avancée, un besoin nettement exprimé du côté des scientifiques eux-mêmes et de la société civile, peu ou pas de concrétisation au niveau institutionnel.

Les scientifiques sont bien conscients des améliorations que l'introduction de la dimension historique peut apporter à la pédagogie des sciences. La baisse des vocations scientifiques dans les pays industrialisés reflète le refus d'une science figée en recettes. L'histoire fait prendre conscience que c'est d'abord une aventure, qui ne sera jamais finie et qui est une des plus belles de l'humanité. D'autre part, l'histoire apprend que la science est faite par des hommes et pour des hommes, qu'elle ne peut être coupée de ses enjeux. Elle fournit aussi un aliment de choix à la réflexion éthique et à l'exercice difficile de l'expertise citoyenne.

Les motions et les travaux théoriques n'ont pas manqué: rapport du prix Nobel Alfred Kastler (1984), de l'European Physical Society (1985), colloque «nouveaux enjeux de l'histoire de la médecine (1990)», journées d'étude de Vigo (1996), de Zagreb (1997), de Bordeaux (1998), journées de pédagogie médicale à Nantes (1999). Un travail considérable, théorique et pratique a été réalisé par la commission Teaching de l'Union: matériaux et expériences 
publiés depuis 1982 par Tea Com News, symposia de Prague (1991), de Zaragoza (1993), d'Istanbul (1995), de Liège (1997).

En revanche, on annonce de plus en plus de suppressions ou de transformations de postes académiques. C'est le cas dans les facultés de médecine, où les postes d'histoire, naguère partie intégrante de l'enseignement médical, sont transformés en chaires de bioéthique ou en chaires purement médicales. La cause en est, de toute évidence, dans l'utilitarisme néo-libéral, qui privilégie l'utilité immédiate des enseignements. On admet bien volontiers que l'histoire des techniques contribue à la formation d'un ingénieur. On préférera cependant un séminaire d'œnologie, puisque l'ingénieur devra organiser des déjeuners d'affaires. Typique est le cas des anciens pays socialistes où l'histoire des sciences s'est effondrée avec le marxisme léninisme qui était sa principale caution. A côté de l'utilitarisme, le mouvement, de plus en plus répandu, de l'anti-science contribue à discréditer nos études.

On se trompe en croyant qu'une stratégie autoritaire résoudra le problème. A supposer même que dans les différents pays une loi impose l'histoire des sciences comme matière obligatoire dans les lycées et les universités, cela ne prouve pas que le contenu du message atteindra véritablement les étudiants et produira un effet.

Notre stratégie est bien plutôt une stratégie de séduction et de communication: connaître les attentes des scientifiques et des sciences humaines, identifier les questions où nos problématiques peuvent apporter du neuf, et y injecter de l'histoire des sciences par micro-doses.

Nous avons trop considéré que le seul vecteur de diffusion de notre discipline était l'enseignement. Or, l'histoire des sciences fait recette à la télévision. Les Science centers, les musées scientifiques, techniques et industriels deviennent des attractions touristiques. Des expositions comme Les ingénieurs de la Renaissance à la Villette attirent des centaines de milliers de personnes. Toutes ces entreprises ont besoin d'historiens des sciences, pas de n'importe lesquels, des historiens qui allient la connaissance approfondie de leur métier, le désir de la vulgariser, la maîtrise des techniques de communication. C'est un nouveau champ d'action qui s'ouvre à nous.

En un siècle d'existence, notre discipline a connu bien des succès et des revers. Son histoire fait penser au mythe grec de Sisyphe, qui roulait un rocher au haut d'une pente. Arrivé au sommet, le rocher dégringolait, et il fallait recommencer. Albert Camus, qui voyait dans Sisyphe l'allégorie de la destinée humaine, concluait son célèbre essai par ces mots: «Il faut imaginer Sisyphe heureux.» 